\title{
System Study: \\ Reactor Core Isolation Cooling \\ 1998-2014
}

\author{
John A. Schroeder
}

December 2015

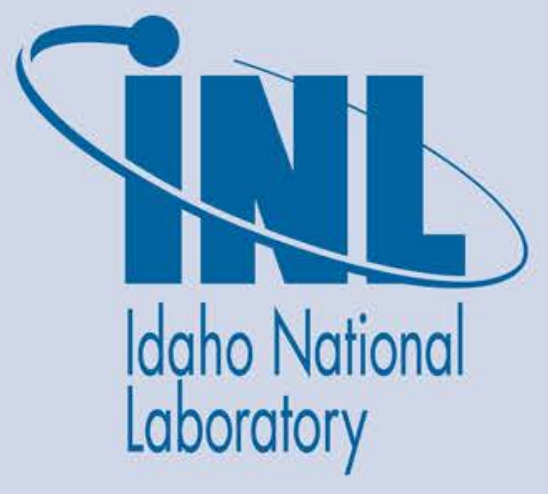

The INL is a U.S. Department of Energy National Laboratory operated by Battelle Energy Alliance 
NOTICE

This information was prepared as an account of work sponsored by an agency of the U.S. Government. Neither the U.S. Government nor any agency thereof, nor any of their employees, makes any warranty, express or implied, or assumes any legal liability or responsibility for any third party's use, or the results of such use, of any information, apparatus, product, or process disclosed herein, or represents that its use by such third party would not infringe privately owned rights. The views expressed herein are not necessarily those of the U.S. Nuclear Regulatory Commission. 


\title{
System Study: Reactor Core Isolation Cooling 1998-2014
}

\author{
John A. Schroeder
}

Update Completed December 2015

\author{
Idaho National Laboratory \\ Risk Assessment and Management Services Department \\ Idaho Falls, Idaho 83415 \\ http://www.inl.gov
}

Prepared for the Division of Risk Assessment Office of Nuclear Regulatory Research

U.S. Nuclear Regulatory Commission NRC Agreement Number NRC-HQ-14-D-0018 



\begin{abstract}
This report presents an unreliability evaluation of the reactor core isolation cooling (RCIC) system at 31 U.S. commercial boiling water reactors. Demand, run hours, and failure data from fiscal year 1998 through 2014 for selected components were obtained from the Institute of Nuclear Power Operations (INPO) Consolidated Events Database (ICES). The unreliability results are trended for the most recent 10-year period while yearly estimates for system unreliability are provided for the entire active period. No statistically significant trends were identified in the RCIC results.
\end{abstract}


System Study

Reactor Core Isolation Cooling
2014 Update December 2015 


\section{CONTENTS}

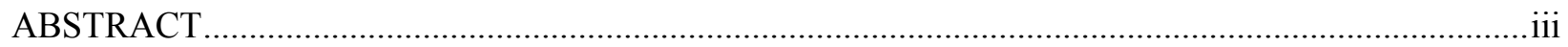

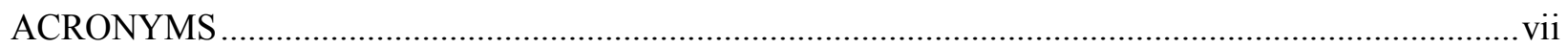

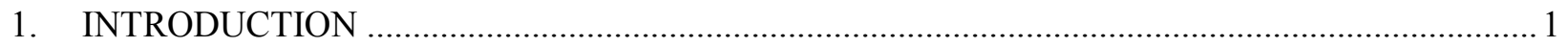

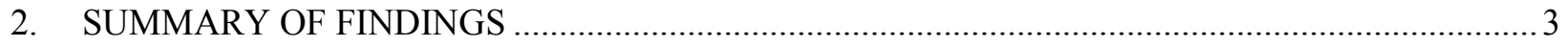

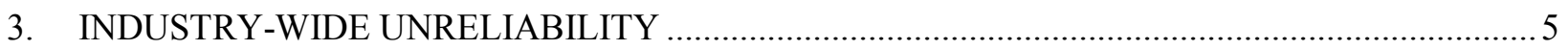

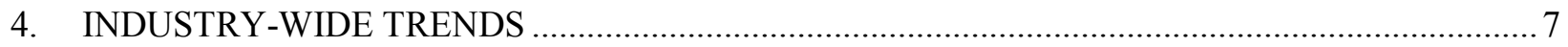

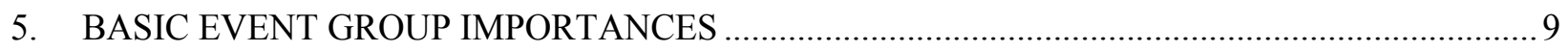

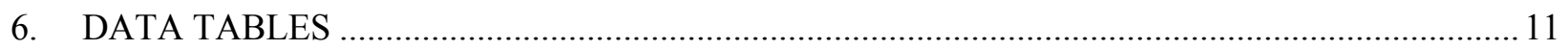

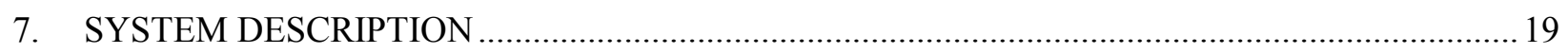

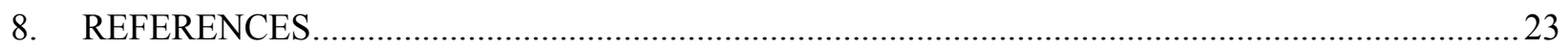

\section{FIGURES}

1. Trend of RCIC system unreliability (start-only model), as a function of fiscal year............................ 8

2. Trend of RCIC system unreliability (8-hour model), as a function of fiscal year. ............................... 8

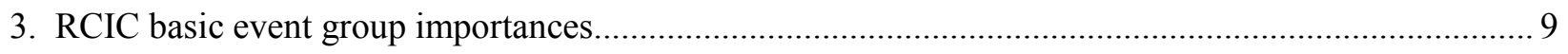

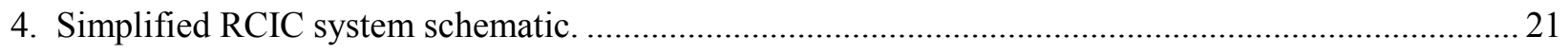

\section{TABLES}

1. BWR plants with a RCIC system selected for the study............................................................. 2

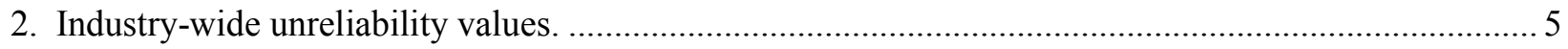

3. RCIC model basic event importance group descriptions............................................................... 10

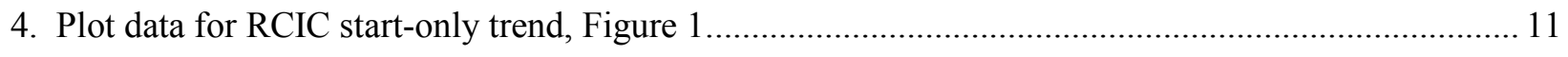

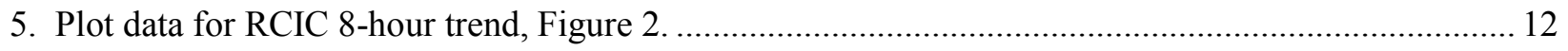




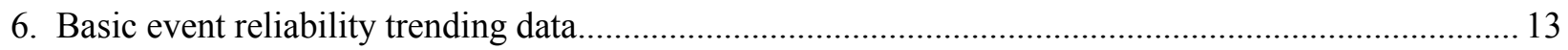

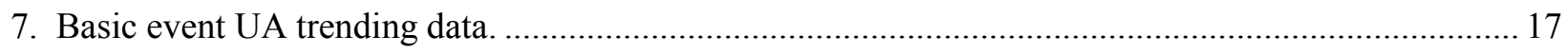

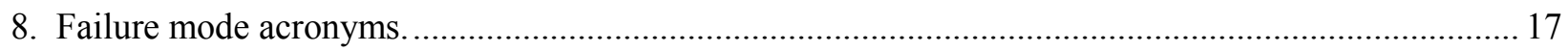




\section{ACRONYMS}

BWR boiling water reactor

CCF common-cause failure

CST condensate storage tank

EPIX Equipment Performance and Information Exchange

FY fiscal year

ICES INPO Consolidated Events Database

INPO Institute of Nuclear Power Operations

MSPI Mitigating Systems Performance Index

PRA probabilistic risk assessment

RCIC reactor core isolation cooling

SPAR standardized plant analysis risk

SSU safety system unavailability 
System Study

Reactor Core Isolation Cooling
2014 Update December 2015 


\section{System Study: Reactor Core Isolation Cooling 1998-2014}

\section{INTRODUCTION}

This report presents an unreliability evaluation of the reactor core isolation cooling (RCIC) systems at the 31 U.S. commercial boiling water reactors (BWRs) (listed in Table 1). For each plant, the corresponding Standardized Plant Analysis Risk (SPAR) model (version model indicated in Table 1) was used in the yearly calculations. Demand, run hours, and failure data from fiscal year (FY)-98 through FY-14 for selected components in the RCIC system were obtained from the Institute of Nuclear Power Operations (INPO) Consolidated Events Database (ICES). Train unavailability data (outages from test or maintenance) were obtained from the Reactor Oversight Process Safety System Unavailability (SSU) database (FY-98 through FY-01) and the Mitigating Systems Performance Index (MSPI) database (FY-02 through FY-14). Common-cause failure (CCF) data used in the models are from the 2010 update to the CCF database. The system unreliability results are trended for the most recent 10 -year period while yearly estimates for system unreliability are provided for the entire active period.

This report does not attempt to estimate basic event values for use in a probabilistic risk assessment (PRA). Suggested values for such use are presented in the 2010 Component Reliability Update

(Reference i), which is an update to Reference ii (NUREG/CR-6928). Baseline RCIC unreliability results using basic event values from that report are summarized in Section 0. Trend results for HPCI (using system-specific data) are presented in Section 4. Similar to previous system study updates, Section 5 contains importance information (using the baseline results from Section 0), and Section 7 describes the RCIC.

The RCIC model is evaluated using the transient flag set in the SPAR model. The transient flag set assumes all support systems are available and that the RCIC system is required to perform to mitigate the effects of the transient initiating event. All models include failures due to unavailability while in test or maintenance. Human error has not been included in the SPAR model logic. An overview of the trending methods, glossary of terms, and abbreviations can be found in the Overview and Reference document on the Reactor Operational Experience Results and Databases web page.

Two modes of the models for the RCIC system are calculated. The RCIC start-only model is the SPAR RCIC model modified by setting all fail-to-run basic events to zero (False), setting all recovery events to False, setting the suction transfer to the suppression pool to False, and setting all cooling basic events to False. The 8-hour mission model includes all basic events in the SPAR RCIC model. 
Table 1. BWR plants with a RCIC system selected for the study.

\begin{tabular}{lc}
\hline \multicolumn{1}{c}{ Plant } & Version \\
\hline Duane Arnold & 8.22 \\
\hline Browns Ferry 1 & 8.22 \\
\hline Browns Ferry 2 & 8.22 \\
\hline Browns Ferry 3 & 8.18 \\
\hline Brunswick 1 & 8.20 \\
\hline Brunswick 2 & 8.20 \\
\hline Clinton 1 & 8.17 \\
\hline Columbia 2 & 8.16 \\
\hline Cooper & 8.22 \\
\hline Fermi 2 & 8.20 \\
\hline FitzPatrick & 8.17 \\
\hline Grand Gulf & 8.22 \\
\hline Hatch 1 & 8.20 \\
\hline Hatch 2 & 8.20 \\
\hline Hope Creek & 8.18 \\
\hline Limerick 1 & 8.20 \\
\hline Limerick 2 & 8.19 \\
\hline La Salle 1 & 8.21 \\
\hline La Salle 2 & 8.21 \\
\hline Monticello & 8.20 \\
\hline Nine Mile Pt. 2 & 8.17 \\
\hline Peach Bottom 2 & 8.25 \\
\hline Peach Bottom 3 & 8.21 \\
\hline Perry & 8.19 \\
\hline Pilgrim & 8.21 \\
\hline Quad Cities 1 & 8.18 \\
\hline Quad Cities 2 & 8.18 \\
\hline River Bend & 8.20 \\
\hline Susquehanna 1 & 8.23 \\
\hline Susquehanna 2 & 8.21 \\
\hline Vermont Yankee & 8.19 \\
\hline
\end{tabular}




\section{SUMMARY OF FINDINGS}

The results of this RCIC system unreliability study are summarized in this section. Of particular interest is the existence of any statistically significant ${ }^{\mathrm{a}}$ increasing trends. In this update, no statistically significant trends were identified in the RCIC unreliability trend results.

The industry-wide RCIC start-only and 8-hour basic event group importances were evaluated and are shown in Figure 3. In both cases, the leading contributor to RCIC system unreliability is the RCIC turbine-driven pump group of basic events. Recovery, restart, and the suppression pool are important to the 8-hour mission model.

a. Statistically significant is defined in terms of the 'p-value.' A p-value is a probability indicating whether to accept or reject the null hypothesis that there is no trend in the data. P-values of less than or equal to 0.05 indicate that we are $95 \%$ confident that there is a trend in the data (reject the null hypothesis of no trend.) By convention, we use the "Michelin Guide" scale: p-value $<0.05$ (statistically significant), p-value $<0.01$ (highly statistically significant); value $<0.001$ (extremely statistically significant). 
System Study

Reactor Core Isolation Cooling
2014 Update December 2015 


\section{INDUSTRY-WIDE UNRELIABILITY}

The RCIC fault trees from the SPAR models were evaluated for each of the 31 operating U.S. commercial boiling water nuclear power plants with a RCIC system.

The industry-wide unreliability of the RCIC system has been estimated for two modes of operation. A failure to start (start-only) model and an 8-hour mission model were evaluated. The uncertainty distributions for RCIC show both plant design variability and parameter uncertainty from the industrywide component failure data (FY-98 through FY-10). ${ }^{a}$

Table 2 shows the percentiles and mean of the aggregated sample data (Latin hypercube, 1000 samples for each model) collected from the uncertainty calculations of the RCIC fault trees in the SPAR models. The lower and upper bounds are based directly on the samples (Latin hypercube) from the uncertainty calculations in the SPAR models. For the industry-level results, the SPAR samples were combined into one large sample in order to determine the industry-level bounds, mean, and median.

Table 2. Industry-wide unreliability values.

\begin{tabular}{ccccc}
\hline Model & $\begin{array}{c}\text { Lower } \\
\mathbf{( 5 \% )}\end{array}$ & Median & Mean & $\begin{array}{c}\text { Upper } \\
\mathbf{( 9 5 \% )}\end{array}$ \\
\hline Start & $1.09 \mathrm{E}-02$ & $2.36 \mathrm{E}-02$ & $2.94 \mathrm{E}-02$ & $6.62 \mathrm{E}-02$ \\
8-hour & $2.59 \mathrm{E}-02$ & $5.11 \mathrm{E}-02$ & $5.72 \mathrm{E}-02$ & $1.08 \mathrm{E}-01$ \\
\hline
\end{tabular}

a. By using industry-wide component failure data, individual plant performance is not included in the distribution of results. 
System Study

Reactor Core Isolation Cooling
2014 Update December 2015 


\section{INDUSTRY-WIDE TRENDS}

The yearly (FY-98 through FY-14) failure and demand or run time data were obtained from EPIX for the RCIC system. RCIC train maintenance unavailability data for trending are from the same time period, as reported in the ROP and EPIX. The component basic event uncertainty was calculated for the RCIC system components using the trending methods described in Section 1 and 2 of the Overview and Reference document. Tables 6 and 7 show the yearly data values for each RCIC system specific component and failure mode combination that was varied in the model. These data were loaded into the RCIC system fault tree in each SPAR model with a RCIC system (see Table 1).

The trend charts show the results of varying component reliability data over time and updating generic, relatively-flat prior distributions using data for each year. In addition, for comparison, the calculated industry-wide system reliability from this update (SPAR/EPIX) is shown. Section 4 of the Overview and Reference link on the System Studies main web page provides more detailed discussion of the trending methods. In the lower left hand corner of the trend figures, the regression method is reported.

The components and failure modes that were varied in the RCIC model are

- RCIC turbine-driven pump start, run, and test and maintenance.

- $\quad$ RCIC injection MOV fail-to-open.

Figure 1 shows the trend in the start-only model unreliability. Table 4 shows the data points for Figure 1. No statistically significant trends within the industry-wide estimates of RCIC system unreliability (start-only) on a per fiscal year basis were identified.

Figure 2 shows the trend in the 8-hour mission unreliability. No statistically significant trend within the industry-wide estimates of RCIC system unreliability (8-hour mission) on a per fiscal year basis was identified. Table 5 shows the data points for Figure 2. 


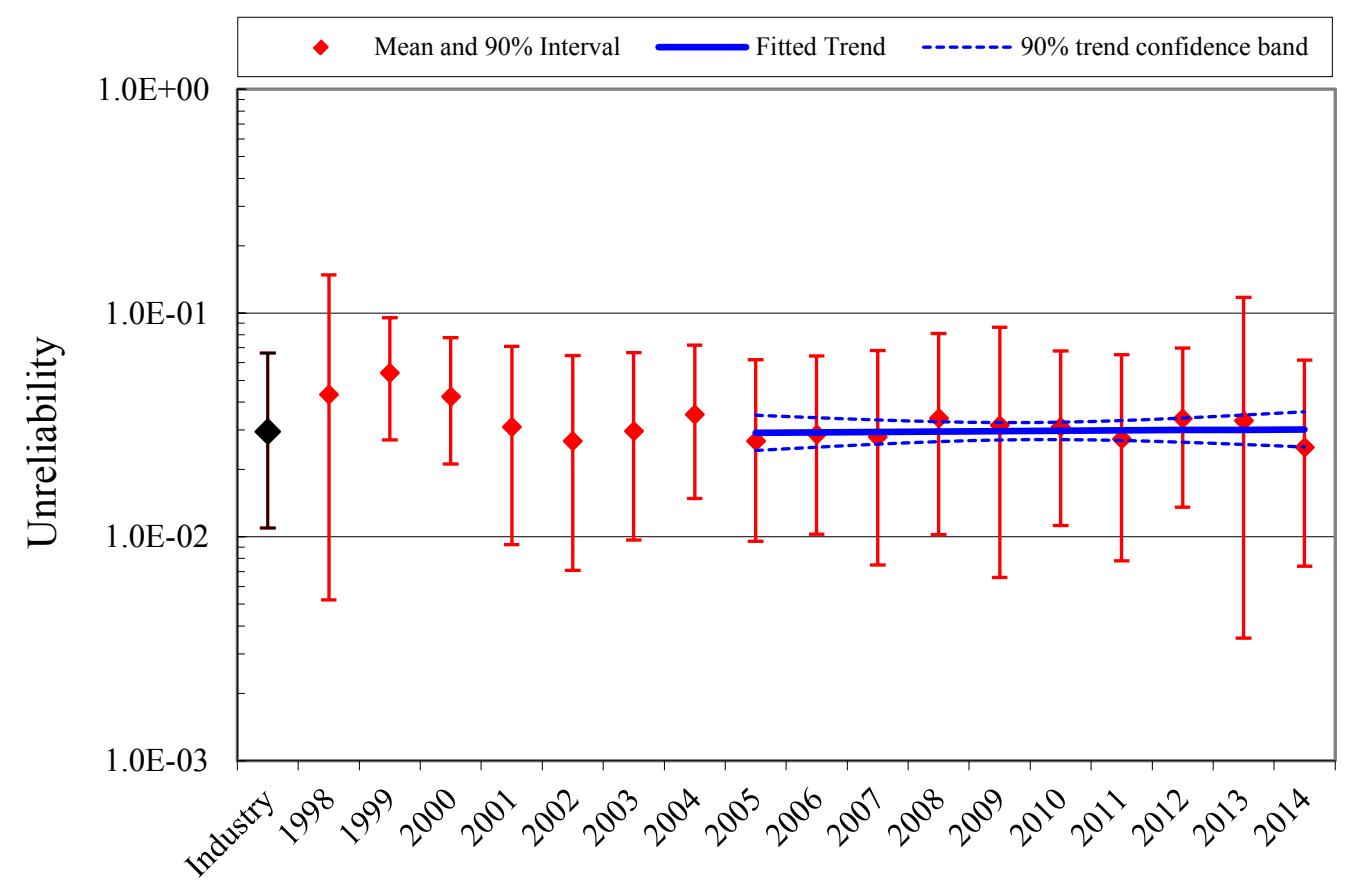

Logit model, $\mathrm{p}$-value $=0.7550 \quad$ Fiscal Year Industry RCIC Start-Only 1-21-2016

Figure 1. Trend of RCIC system unreliability (start-only model), as a function of fiscal year.

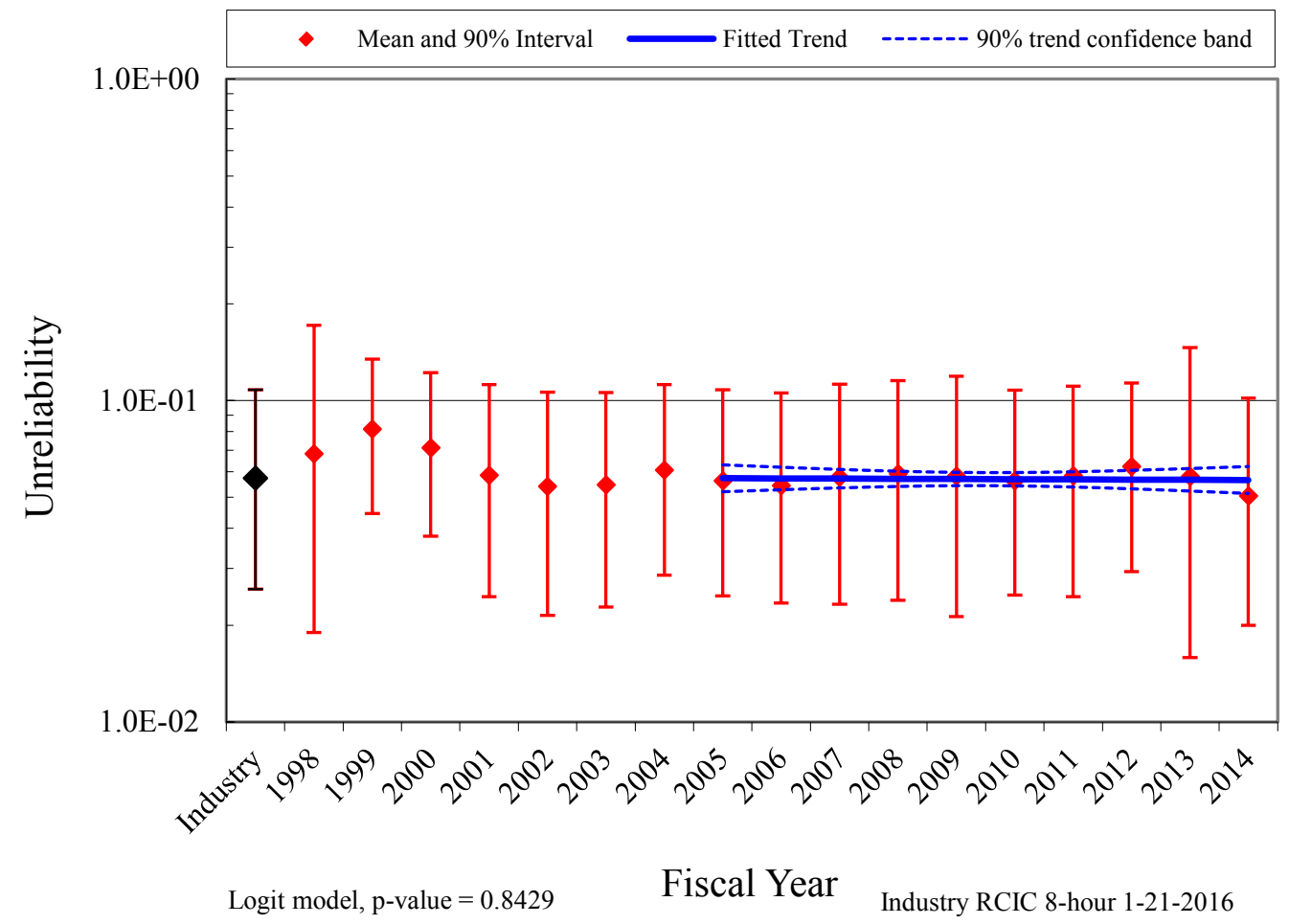

Figure 2. Trend of RCIC system unreliability (8-hour model), as a function of fiscal year. 


\section{BASIC EVENT GROUP IMPORTANCES}

The RCIC basic event group Fussell-Vesely importances were calculated for the start-only and 8-hour modes for each plant using the industry-wide data (1998-2010). These basic event group importances were then averaged across all plants to represent an industry-wide basic event group importance. The industry-wide RCIC start-only and 8-hour basic event group importances are shown in Figure 3.

In both cases, the leading contributor to RCIC system unreliability is the RCIC turbine-driven pump group of basic events. Recovery, restart, and the suppression pool are important to the 8-hour mission model. For more discussion on the RCIC turbine-driven pumps, see the turbine-driven pump component reliability study at NRC Reactor Operational Experience Results and Databases web site. Table 3 shows the SPAR model RCIC importance groups and their descriptions.

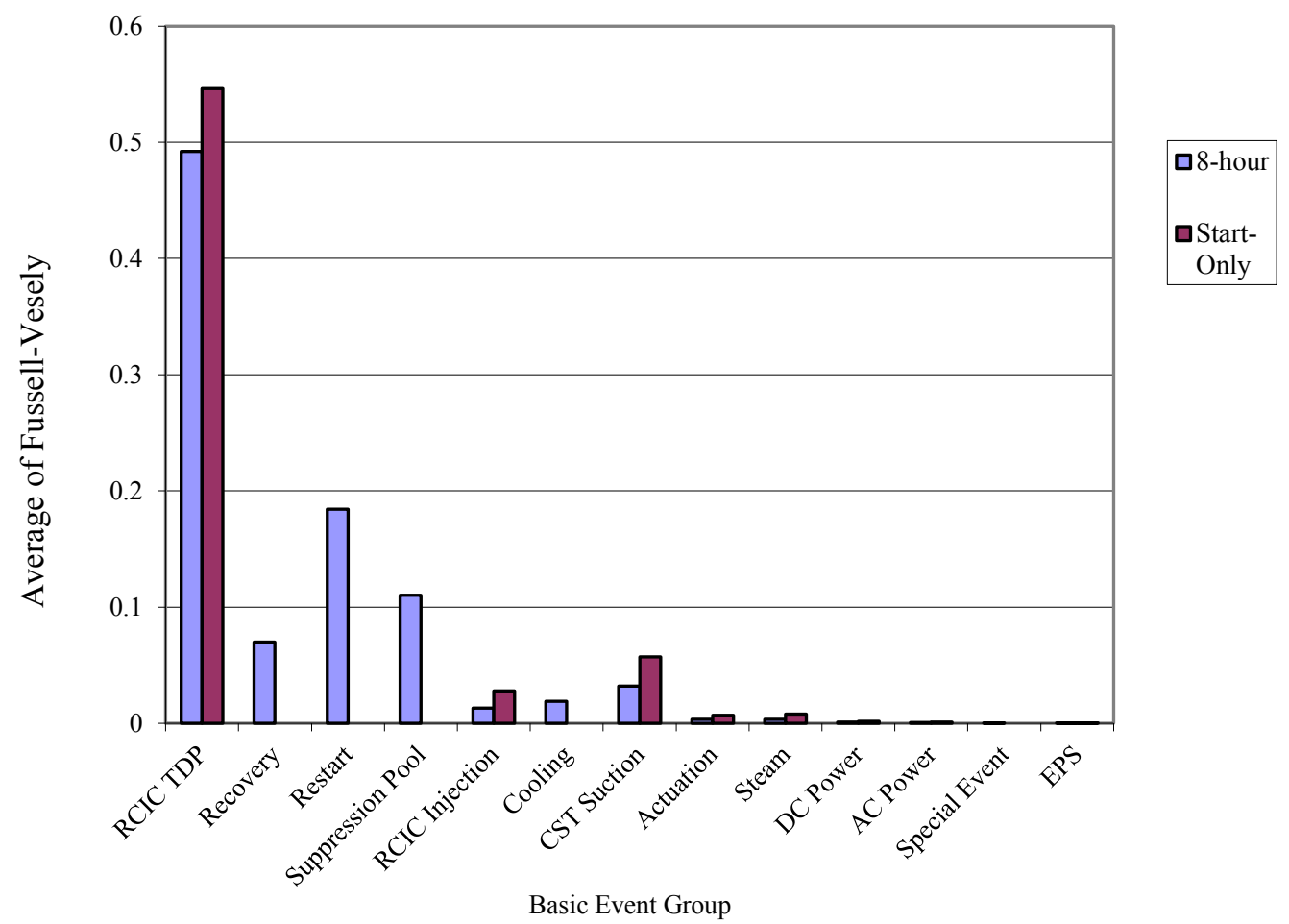

Figure 3. RCIC basic event group importances. 
Table 3. RCIC model basic event importance group descriptions.

Group

AC Power

Actuation

Cooling

CST Suction

DC Power

EPS

$\mathrm{RCIC}$ Injection

RCIC TDP

Recovery

Restart

Special Event

The ac buses and circuit breakers that supply power to the service water pumps and motor-operated valves.

ESF actuation circuitry.

The pumps, valves, and heat exchangers that provide heat removal to the $\mathrm{RCIC}$ turbine-driven pump and the $\mathrm{RCIC}$ room.

The motor-operated valves and air-operated valves in the condensate storage tank suction path. Includes the failure of the condensate storage tank.

The batteries and battery chargers that supply power to the RCIC turbine-driven pump control circuitry.

$\mathrm{RCIC}$ dependency on the emergency power system.

The motor-operated valves and check valves in the $\mathrm{RCIC}$ injection path.

All basic events associated with the turbine-driven pumps. The start, run, common-cause, and test and maintenance are included in this group of basic events.

The operator recovery of the TDP FTS, FTR, and other specialized modeled recovery events.

The required restart of the $\mathrm{RCIC}$ turbine-driven pump. Includes the probability that restart is required and the probability that the turbine-driven pump will restart.

Various events used in the models that are not directly associated with the RCIC system.

Steam The turbine steam supply valves.

Suppression Pool The suppression pool motor-operated valves, check valves, and strainers required when a need to transfer to the suppression pool (torus) occurs. 


\section{DATA TABLES}

Table 4. Plot data for RCIC start-only trend, Figure 1.

\begin{tabular}{ccccccc}
\hline & \multicolumn{2}{c}{ Regression Curve Data Points } & \multicolumn{2}{c}{ Annual Estimate Data Points } \\
\cline { 2 - 6 } FY/Source & Mean & $\begin{array}{c}\text { Lower } \\
\mathbf{( 5 \% )}\end{array}$ & $\begin{array}{c}\text { Upper } \\
\mathbf{( 9 5 \% )}\end{array}$ & $\begin{array}{c}\text { Lower } \\
\mathbf{( 5 \% )}\end{array}$ & $\begin{array}{c}\text { Upper } \\
\mathbf{( 9 5 \% )}\end{array}$ & Mean \\
\hline SPAR/ICES & & & & $1.09 \mathrm{E}-02$ & $6.62 \mathrm{E}-02$ & $2.94 \mathrm{E}-02$ \\
1998 & & & & $5.22 \mathrm{E}-03$ & $1.48 \mathrm{E}-01$ & $4.32 \mathrm{E}-02$ \\
1999 & & & & $2.71 \mathrm{E}-02$ & $9.55 \mathrm{E}-02$ & $5.38 \mathrm{E}-02$ \\
\hline 2000 & & & & $2.12 \mathrm{E}-02$ & $7.77 \mathrm{E}-02$ & $4.22 \mathrm{E}-02$ \\
\hline 2001 & & & & $9.24 \mathrm{E}-03$ & $7.09 \mathrm{E}-02$ & $3.08 \mathrm{E}-02$ \\
\hline 2002 & & & & $7.09 \mathrm{E}-03$ & $6.46 \mathrm{E}-02$ & $2.67 \mathrm{E}-02$ \\
\hline 2003 & & & & $9.68 \mathrm{E}-03$ & $6.64 \mathrm{E}-02$ & $2.95 \mathrm{E}-02$ \\
\hline 2004 & & & & $1.48 \mathrm{E}-02$ & $7.19 \mathrm{E}-02$ & $3.52 \mathrm{E}-02$ \\
\hline 2005 & $2.92 \mathrm{E}-02$ & $2.43 \mathrm{E}-02$ & $3.50 \mathrm{E}-02$ & $9.54 \mathrm{E}-03$ & $6.20 \mathrm{E}-02$ & $2.68 \mathrm{E}-02$ \\
\hline 2006 & $2.93 \mathrm{E}-02$ & $2.52 \mathrm{E}-02$ & $3.41 \mathrm{E}-02$ & $1.03 \mathrm{E}-02$ & $6.44 \mathrm{E}-02$ & $2.87 \mathrm{E}-02$ \\
\hline 2007 & $2.94 \mathrm{E}-02$ & $2.59 \mathrm{E}-02$ & $3.33 \mathrm{E}-02$ & $7.50 \mathrm{E}-03$ & $6.81 \mathrm{E}-02$ & $2.79 \mathrm{E}-02$ \\
\hline 2008 & $2.95 \mathrm{E}-02$ & $2.66 \mathrm{E}-02$ & $3.27 \mathrm{E}-02$ & $1.02 \mathrm{E}-02$ & $8.10 \mathrm{E}-02$ & $3.39 \mathrm{E}-02$ \\
\hline 2009 & $2.96 \mathrm{E}-02$ & $2.71 \mathrm{E}-02$ & $3.24 \mathrm{E}-02$ & $6.58 \mathrm{E}-03$ & $8.63 \mathrm{E}-02$ & $3.14 \mathrm{E}-02$ \\
\hline 2010 & $2.97 \mathrm{E}-02$ & $2.72 \mathrm{E}-02$ & $3.25 \mathrm{E}-02$ & $1.12 \mathrm{E}-02$ & $6.78 \mathrm{E}-02$ & $3.09 \mathrm{E}-02$ \\
\hline 2011 & $2.99 \mathrm{E}-02$ & $2.70 \mathrm{E}-02$ & $3.31 \mathrm{E}-02$ & $7.83 \mathrm{E}-03$ & $6.52 \mathrm{E}-02$ & $2.73 \mathrm{E}-02$ \\
\hline 2012 & $3.00 \mathrm{E}-02$ & $2.65 \mathrm{E}-02$ & $3.40 \mathrm{E}-02$ & $1.35 \mathrm{E}-02$ & $6.97 \mathrm{E}-02$ & $3.36 \mathrm{E}-02$ \\
\hline 2013 & $3.01 \mathrm{E}-02$ & $2.59 \mathrm{E}-02$ & $3.50 \mathrm{E}-02$ & $3.53 \mathrm{E}-03$ & $1.18 \mathrm{E}-01$ & $3.30 \mathrm{E}-02$ \\
\hline 2014 & $3.02 \mathrm{E}-02$ & $2.52 \mathrm{E}-02$ & $3.62 \mathrm{E}-02$ & $7.38 \mathrm{E}-03$ & $6.18 \mathrm{E}-02$ & $2.50 \mathrm{E}-02$ \\
\hline
\end{tabular}


Table 5. Plot data for RCIC 8-hour trend, Figure 2.

\begin{tabular}{ccccccc}
\hline & \multicolumn{2}{c}{ Regression Curve Data Points } & \multicolumn{2}{c}{ Plot Trend Error Bar Points } \\
\cline { 2 - 6 } FY/Source & Mean & $\begin{array}{c}\text { Lower } \\
\mathbf{( 5 \% )}\end{array}$ & $\begin{array}{c}\text { Upper } \\
\mathbf{( 9 5 \% )}\end{array}$ & $\begin{array}{c}\text { Lower } \\
\mathbf{( 5 \% )}\end{array}$ & $\begin{array}{c}\text { Upper } \\
\mathbf{( 9 5 \% )}\end{array}$ & Mean \\
\hline SPAR/ICES & & & & $2.59 \mathrm{E}-02$ & $1.08 \mathrm{E}-01$ & $5.72 \mathrm{E}-02$ \\
1998 & & & & $1.90 \mathrm{E}-02$ & $1.71 \mathrm{E}-01$ & $6.81 \mathrm{E}-02$ \\
\hline 1999 & & & & $4.45 \mathrm{E}-02$ & $1.35 \mathrm{E}-01$ & $8.14 \mathrm{E}-02$ \\
\hline 2000 & & & & $3.78 \mathrm{E}-02$ & $1.22 \mathrm{E}-01$ & $7.12 \mathrm{E}-02$ \\
\hline 2001 & & & & $2.45 \mathrm{E}-02$ & $1.12 \mathrm{E}-01$ & $5.85 \mathrm{E}-02$ \\
2002 & & & & $2.14 \mathrm{E}-02$ & $1.06 \mathrm{E}-01$ & $5.39 \mathrm{E}-02$ \\
\hline 2003 & & & & $2.28 \mathrm{E}-02$ & $1.06 \mathrm{E}-01$ & $5.46 \mathrm{E}-02$ \\
\hline 2004 & & & & $2.86 \mathrm{E}-02$ & $1.12 \mathrm{E}-01$ & $6.06 \mathrm{E}-02$ \\
\hline 2005 & $5.73 \mathrm{E}-02$ & $5.20 \mathrm{E}-02$ & $6.31 \mathrm{E}-02$ & $2.47 \mathrm{E}-02$ & $1.08 \mathrm{E}-01$ & $5.62 \mathrm{E}-02$ \\
\hline 2006 & $5.72 \mathrm{E}-02$ & $5.28 \mathrm{E}-02$ & $6.20 \mathrm{E}-02$ & $2.35 \mathrm{E}-02$ & $1.05 \mathrm{E}-01$ & $5.43 \mathrm{E}-02$ \\
\hline 2007 & $5.71 \mathrm{E}-02$ & $5.35 \mathrm{E}-02$ & $6.10 \mathrm{E}-02$ & $2.32 \mathrm{E}-02$ & $1.12 \mathrm{E}-01$ & $5.77 \mathrm{E}-02$ \\
\hline 2008 & $5.71 \mathrm{E}-02$ & $5.40 \mathrm{E}-02$ & $6.03 \mathrm{E}-02$ & $2.39 \mathrm{E}-02$ & $1.15 \mathrm{E}-01$ & $5.91 \mathrm{E}-02$ \\
\hline 2009 & $5.70 \mathrm{E}-02$ & $5.43 \mathrm{E}-02$ & $5.98 \mathrm{E}-02$ & $2.13 \mathrm{E}-02$ & $1.19 \mathrm{E}-01$ & $5.81 \mathrm{E}-02$ \\
\hline 2010 & $5.69 \mathrm{E}-02$ & $5.43 \mathrm{E}-02$ & $5.97 \mathrm{E}-02$ & $2.48 \mathrm{E}-02$ & $1.08 \mathrm{E}-01$ & $5.61 \mathrm{E}-02$ \\
\hline 2011 & $5.68 \mathrm{E}-02$ & $5.38 \mathrm{E}-02$ & $6.00 \mathrm{E}-02$ & $2.45 \mathrm{E}-02$ & $1.11 \mathrm{E}-01$ & $5.83 \mathrm{E}-02$ \\
\hline 2012 & $5.68 \mathrm{E}-02$ & $5.31 \mathrm{E}-02$ & $6.06 \mathrm{E}-02$ & $2.94 \mathrm{E}-02$ & $1.13 \mathrm{E}-01$ & $6.21 \mathrm{E}-02$ \\
\hline 2013 & $5.67 \mathrm{E}-02$ & $5.23 \mathrm{E}-02$ & $6.14 \mathrm{E}-02$ & $1.58 \mathrm{E}-02$ & $1.46 \mathrm{E}-01$ & $5.80 \mathrm{E}-02$ \\
\hline 2014 & $5.66 \mathrm{E}-02$ & $5.14 \mathrm{E}-02$ & $6.23 \mathrm{E}-02$ & $2.00 \mathrm{E}-02$ & $1.02 \mathrm{E}-01$ & $5.03 \mathrm{E}-02$ \\
\hline
\end{tabular}


Table 6. Basic event reliability trending data.

\begin{tabular}{|c|c|c|c|c|c|c|c|c|}
\hline \multirow{2}{*}{$\begin{array}{l}\text { Failure } \\
\text { Mode }\end{array}$} & \multirow[b]{2}{*}{ Component } & \multirow[b]{2}{*}{ Year } & \multirow{2}{*}{$\begin{array}{l}\text { Number of } \\
\text { Failures }\end{array}$} & \multirow{2}{*}{$\begin{array}{l}\text { Demands/ } \\
\text { Run Hours }\end{array}$} & \multicolumn{4}{|c|}{ Bayesian Update } \\
\hline & & & & & Mean & Post A & Post B & Distribution \\
\hline FTOC & AOV & 1998 & 0 & 71.3 & 8.96E-04 & 1.112 & 1239 & Beta \\
\hline FTOC & AOV & 1999 & 0 & 75.4 & 8.94E-04 & 1.112 & 1243 & Beta \\
\hline FTOC & AOV & 2000 & 0 & 75.4 & 8.94E-04 & 1.112 & 1243 & Beta \\
\hline FTOC & AOV & 2001 & 0 & 75.4 & 8.94E-04 & 1.112 & 1243 & Beta \\
\hline FTOC & AOV & 2002 & 0 & 75.4 & 8.94E-04 & 1.112 & 1243 & Beta \\
\hline FTOC & AOV & 2003 & 0 & 75.1 & 8.94E-04 & 1.112 & 1243 & Beta \\
\hline FTOC & AOV & 2004 & 0 & 75.4 & 8.94E-04 & 1.112 & 1243 & Beta \\
\hline FTOC & AOV & 2005 & 0 & 75.4 & 8.94E-04 & 1.112 & 1243 & Beta \\
\hline FTOC & AOV & 2006 & 0 & 73.0 & 8.95E-04 & 1.112 & 1241 & Beta \\
\hline FTOC & AOV & 2007 & 0 & 71.7 & 8.96E-04 & 1.112 & 1240 & Beta \\
\hline FTOC & AOV & 2008 & 1 & 74.8 & 1.70E-03 & 2.112 & 1242 & Beta \\
\hline FTOC & AOV & 2009 & 0 & 76.2 & 8.93E-04 & 1.112 & 1244 & Beta \\
\hline FTOC & AOV & 2010 & 0 & 76.2 & 8.93E-04 & 1.112 & 1244 & Beta \\
\hline FTOC & AOV & 2011 & 0 & 76.2 & 8.93E-04 & 1.112 & 1244 & Beta \\
\hline FTOC & AOV & 2012 & 0 & 76.2 & 8.93E-04 & 1.112 & 1244 & Beta \\
\hline FTOC & AOV & 2013 & 0 & 76.2 & 8.93E-04 & 1.112 & 1244 & Beta \\
\hline FTOC & AOV & 2014 & 0 & 76.2 & 8.93E-04 & 1.112 & 1244 & Beta \\
\hline FTOC & MOV & 1998 & 3 & 2379.0 & 1.12E-03 & 5.046 & 4499 & Beta \\
\hline FTOC & MOV & 1999 & 7 & 2498.2 & 1.96E-03 & 9.046 & 4614 & Beta \\
\hline FTOC & MOV & 2000 & 4 & 2520.8 & 1.30E-03 & 6.046 & 4640 & Beta \\
\hline FTOC & MOV & 2001 & 5 & 2707.3 & 1.46E-03 & 7.046 & 4825 & Beta \\
\hline FTOC & MOV & 2002 & 3 & 2789.1 & 1.03E-03 & 5.046 & 4909 & Beta \\
\hline FTOC & MOV & 2003 & 2 & 3400.6 & 7.32E-04 & 4.046 & 5522 & Beta \\
\hline FTOC & MOV & 2004 & 2 & 2601.8 & 8.56E-04 & 4.046 & 4723 & Beta \\
\hline FTOC & MOV & 2005 & 4 & 2675.2 & 1.26E-03 & 6.046 & 4794 & Beta \\
\hline FTOC & MOV & 2006 & 3 & 2648.5 & 1.06E-03 & 5.046 & 4769 & Beta \\
\hline FTOC & MOV & 2007 & 3 & 2708.0 & 1.04E-03 & 5.046 & 4828 & Beta \\
\hline FTOC & MOV & 2008 & 2 & 2779.6 & 8.25E-04 & 4.046 & 4901 & Beta \\
\hline FTOC & MOV & 2009 & 2 & 2670.7 & 8.44E-04 & 4.046 & 4792 & Beta \\
\hline FTOC & MOV & 2010 & 1 & 2637.6 & $6.40 \mathrm{E}-04$ & 3.046 & 4760 & Beta \\
\hline FTOC & MOV & 2011 & 2 & 2605.3 & 8.55E-04 & 4.046 & 4726 & Beta \\
\hline FTOC & MOV & 2012 & 5 & 2683.9 & 1.47E-03 & 7.046 & 4802 & Beta \\
\hline FTOC & MOV & 2013 & 1 & 2868.8 & $6.10 \mathrm{E}-04$ & 3.046 & 4991 & Beta \\
\hline FTOC & MOV & 2014 & 2 & 2613.5 & 8.54E-04 & 4.046 & 4734 & Beta \\
\hline FTOP & AOV & 1998 & 0 & 61320.0 & 2.46E-07 & 1.421 & 5780320 & Gamma \\
\hline FTOP & AOV & 1999 & 0 & 61320.0 & 2.46E-07 & 1.421 & 5780320 & Gamma \\
\hline FTOP & AOV & 2000 & 0 & 61320.0 & 2.46E-07 & 1.421 & 5780320 & Gamma \\
\hline FTOP & AOV & 2001 & 0 & 61320.0 & 2.46E-07 & 1.421 & 5780320 & Gamma \\
\hline FTOP & AOV & 2002 & 0 & 61320.0 & 2.46E-07 & 1.421 & 5780320 & Gamma \\
\hline FTOP & AOV & 2003 & 0 & 61320.0 & 2.46E-07 & 1.421 & 5780320 & Gamma \\
\hline FTOP & AOV & 2004 & 0 & 61320.0 & 2.46E-07 & 1.421 & 5780320 & Gamma \\
\hline
\end{tabular}


Table 6. (continued).

\begin{tabular}{|c|c|c|c|c|c|c|c|c|}
\hline \multirow{2}{*}{$\begin{array}{l}\text { Failure } \\
\text { Mode }\end{array}$} & \multirow[b]{2}{*}{ Component } & \multirow[b]{2}{*}{ Year } & \multirow{2}{*}{$\begin{array}{l}\text { Number of } \\
\text { Failures }\end{array}$} & \multirow{2}{*}{$\begin{array}{l}\text { Demands/ } \\
\text { Run Hours }\end{array}$} & \multicolumn{4}{|c|}{ Bayesian Update } \\
\hline & & & & & Mean & Post A & Post B & Distribution \\
\hline FTOP & AOV & 2005 & 0 & 61320.0 & $2.46 \mathrm{E}-07$ & 1.421 & 5780320 & Gamma \\
\hline FTOP & AOV & 2006 & 0 & 61320.0 & 2.46E-07 & 1.421 & 5780320 & Gamma \\
\hline FTOP & AOV & 2007 & 0 & 52560.0 & 2.46E-07 & 1.421 & 5771560 & Gamma \\
\hline FTOP & AOV & 2008 & 0 & 52560.0 & 2.46E-07 & 1.421 & 5771560 & Gamma \\
\hline FTOP & AOV & 2009 & 0 & 52560.0 & 2.46E-07 & 1.421 & 5771560 & Gamma \\
\hline FTOP & AOV & 2010 & 0 & 52560.0 & 2.46E-07 & 1.421 & 5771560 & Gamma \\
\hline FTOP & AOV & 2011 & 0 & 52560.0 & 2.46E-07 & 1.421 & 5771560 & Gamma \\
\hline FTOP & AOV & 2012 & 1 & 52560.0 & 4.19E-07 & 2.421 & 5771560 & Gamma \\
\hline FTOP & AOV & 2013 & 0 & 52560.0 & $2.46 \mathrm{E}-07$ & 1.421 & 5771560 & Gamma \\
\hline FTOP & AOV & 2014 & 0 & 52560.0 & 2.46E-07 & 1.421 & 5771560 & Gamma \\
\hline FTOP & MOV & 1998 & 0 & 2522880.0 & 5.93E-08 & 1.458 & 24572880 & Gamma \\
\hline FTOP & MOV & 1999 & 2 & 2549160.0 & $1.41 \mathrm{E}-07$ & 3.458 & 24599160 & Gamma \\
\hline FTOP & MOV & 2000 & 0 & 2575440.0 & 5.92E-08 & 1.458 & 24625440 & Gamma \\
\hline FTOP & MOV & 2001 & 0 & 2549160.0 & 5.93E-08 & 1.458 & 24599160 & Gamma \\
\hline FTOP & MOV & 2002 & 0 & 2549160.0 & 5.93E-08 & 1.458 & 24599160 & Gamma \\
\hline FTOP & MOV & 2003 & 0 & 2549160.0 & 5.93E-08 & 1.458 & 24599160 & Gamma \\
\hline FTOP & MOV & 2004 & 0 & 2549160.0 & 5.93E-08 & 1.458 & 24599160 & Gamma \\
\hline FTOP & MOV & 2005 & 0 & 2549160.0 & 5.93E-08 & 1.458 & 24599160 & Gamma \\
\hline FTOP & MOV & 2006 & 0 & 2566680.0 & 5.92E-08 & 1.458 & 24616680 & Gamma \\
\hline FTOP & MOV & 2007 & 0 & 2566680.0 & 5.92E-08 & 1.458 & 24616680 & Gamma \\
\hline FTOP & MOV & 2008 & 0 & 2566680.0 & 5.92E-08 & 1.458 & 24616680 & Gamma \\
\hline FTOP & MOV & 2009 & 0 & 2566680.0 & 5.92E-08 & 1.458 & 24616680 & Gamma \\
\hline FTOP & MOV & 2010 & 0 & 2654280.0 & 5.90E-08 & 1.458 & 24704280 & Gamma \\
\hline FTOP & MOV & 2011 & 0 & 2645520.0 & 5.90E-08 & 1.458 & 24695520 & Gamma \\
\hline FTOP & MOV & 2012 & 0 & 2592960.0 & 5.92E-08 & 1.458 & 24642960 & Gamma \\
\hline FTOP & MOV & 2013 & 0 & 2592960.0 & 5.92E-08 & 1.458 & 24642960 & Gamma \\
\hline FTOP & MOV & 2014 & 0 & 2584200.0 & 5.92E-08 & 1.458 & 24634200 & Gamma \\
\hline $\mathrm{FTR}<1 \mathrm{H}$ & TDP & 1998 & 0 & 241.5 & 2.10E-03 & 0.9618 & 458 & Gamma \\
\hline $\mathrm{FTR}<1 \mathrm{H}$ & TDP & 1999 & 1 & 239.4 & 4.30E-03 & 1.9618 & 456 & Gamma \\
\hline $\mathrm{FTR}<1 \mathrm{H}$ & TDP & 2000 & 2 & 279.3 & 5.98E-03 & 2.9618 & 496 & Gamma \\
\hline $\mathrm{FTR}<1 \mathrm{H}$ & TDP & 2001 & 1 & 283.1 & 3.93E-03 & 1.9618 & 500 & Gamma \\
\hline $\mathrm{FTR}<1 \mathrm{H}$ & TDP & 2002 & 1 & 291.7 & 3.86E-03 & 1.9618 & 508 & Gamma \\
\hline $\mathrm{FTR}<1 \mathrm{H}$ & TDP & 2003 & 0 & 365.0 & 1.65E-03 & 0.9618 & 581 & Gamma \\
\hline $\mathrm{FTR}<1 \mathrm{H}$ & TDP & 2004 & 0 & 282.1 & 1.93E-03 & 0.9618 & 498 & Gamma \\
\hline $\mathrm{FTR}<1 \mathrm{H}$ & TDP & 2005 & 2 & 289.5 & 5.85E-03 & 2.9618 & 506 & Gamma \\
\hline $\mathrm{FTR}<1 \mathrm{H}$ & TDP & 2006 & 0 & 267.9 & 1.99E-03 & 0.9618 & 484 & Gamma \\
\hline $\mathrm{FTR}<1 \mathrm{H}$ & TDP & 2007 & 2 & 284.5 & 5.91E-03 & 2.9618 & 501 & Gamma \\
\hline $\mathrm{FTR}<1 \mathrm{H}$ & TDP & 2008 & 0 & 261.6 & 2.01E-03 & 0.9618 & 478 & Gamma \\
\hline $\mathrm{FTR}<1 \mathrm{H}$ & TDP & 2009 & 1 & 260.5 & 4.11E-03 & 1.9618 & 477 & Gamma \\
\hline $\mathrm{FTR}<1 \mathrm{H}$ & TDP & 2010 & 0 & 266.2 & 1.99E-03 & 0.9618 & 483 & Gamma \\
\hline $\mathrm{FTR}<1 \mathrm{H}$ & TDP & 2011 & 3 & 269.0 & 8.16E-03 & 3.9618 & 485 & Gamma \\
\hline
\end{tabular}


Table 6. (continued).

\begin{tabular}{|c|c|c|c|c|c|c|c|c|}
\hline \multirow{2}{*}{$\begin{array}{l}\text { Failure } \\
\text { Mode }\end{array}$} & \multirow[b]{2}{*}{ Component } & \multirow[b]{2}{*}{ Year } & \multirow{2}{*}{$\begin{array}{l}\text { Number of } \\
\text { Failures }\end{array}$} & \multirow{2}{*}{$\begin{array}{l}\text { Demands/ } \\
\text { Run Hours }\end{array}$} & \multicolumn{4}{|c|}{ Bayesian Update } \\
\hline & & & & & Mean & Post A & Post B & Distribution \\
\hline $\mathrm{FTR}<1 \mathrm{H}$ & TDP & 2012 & 1 & 269.1 & 4.04E-03 & 1.9618 & 486 & Gamma \\
\hline $\mathrm{FTR}<1 \mathrm{H}$ & TDP & 2013 & 0 & 300.8 & 1.86E-03 & 0.9618 & 517 & Gamma \\
\hline $\mathrm{FTR}<1 \mathrm{H}$ & TDP & 2014 & 0 & 273.7 & 1.96E-03 & 0.9618 & 490 & Gamma \\
\hline FTR $>1 \mathrm{H}$ & TDP & 1998 & 1 & 54.6 & 1.67E-03 & 13.5 & 8,082 & Gamma \\
\hline $\mathrm{FTR}>1 \mathrm{H}$ & TDP & 1999 & 0 & 64.2 & $1.54 \mathrm{E}-03$ & 12.5 & 8,092 & Gamma \\
\hline FTR $>1 \mathrm{H}$ & TDP & 2000 & 0 & 47.0 & $1.55 \mathrm{E}-03$ & 12.5 & 8,075 & Gamma \\
\hline FTR $>1 \mathrm{H}$ & TDP & 2001 & 0 & 157.3 & $1.53 \mathrm{E}-03$ & 12.5 & 8,185 & Gamma \\
\hline FTR $>1 \mathrm{H}$ & TDP & 2002 & 0 & 54.0 & $1.55 \mathrm{E}-03$ & 12.5 & 8,082 & Gamma \\
\hline FTR $>1 \mathrm{H}$ & TDP & 2003 & 0 & 86.8 & $1.54 \mathrm{E}-03$ & 12.5 & 8,114 & Gamma \\
\hline FTR $>1 \mathrm{H}$ & TDP & 2004 & 0 & 33.2 & $1.55 \mathrm{E}-03$ & 12.5 & 8,061 & Gamma \\
\hline FTR $>1 \mathrm{H}$ & TDP & 2005 & 0 & 34.7 & $1.55 \mathrm{E}-03$ & 12.5 & 8,062 & Gamma \\
\hline $\mathrm{FTR}>1 \mathrm{H}$ & TDP & 2006 & 0 & 41.7 & $1.55 \mathrm{E}-03$ & 12.5 & 8,069 & Gamma \\
\hline $\mathrm{FTR}>1 \mathrm{H}$ & TDP & 2007 & 1 & 38.9 & 1.67E-03 & 13.5 & 8,067 & Gamma \\
\hline FTR $>1 \mathrm{H}$ & TDP & 2008 & 0 & 57.0 & $1.55 \mathrm{E}-03$ & 12.5 & 8,085 & Gamma \\
\hline $\mathrm{FTR}>1 \mathrm{H}$ & TDP & 2009 & 0 & 35.8 & 1.55E-03 & 12.5 & 8,064 & Gamma \\
\hline FTR $>1 \mathrm{H}$ & TDP & 2010 & 0 & 74.9 & 1.54E-03 & 12.5 & 8,103 & Gamma \\
\hline FTR $>1 \mathrm{H}$ & TDP & 2011 & 0 & 35.4 & $1.55 \mathrm{E}-03$ & 12.5 & 8,063 & Gamma \\
\hline FTR $>1 \mathrm{H}$ & TDP & 2012 & 1 & 71.7 & 1.67E-03 & 13.5 & 8,099 & Gamma \\
\hline $\mathrm{FTR}>1 \mathrm{H}$ & TDP & 2013 & 1 & 115.3 & 1.66E-03 & 13.5 & 8,143 & Gamma \\
\hline $\mathrm{FTR}>1 \mathrm{H}$ & TDP & 2014 & 0 & 59.1 & $1.55 \mathrm{E}-03$ & 12.5 & 8,087 & Gamma \\
\hline FTS & TDP & 1998 & 1 & 241.5 & 5.02E-03 & 1.9421 & 385 & Beta \\
\hline FTS & TDP & 1999 & 9 & 239.4 & 2.59E-02 & 9.9421 & 375 & Beta \\
\hline FTS & TDP & 2000 & 7 & 279.3 & 1.87E-02 & 7.9421 & 416 & Beta \\
\hline FTS & TDP & 2001 & 2 & 283.1 & 6.87E-03 & 2.9421 & 425 & Beta \\
\hline FTS & TDP & 2002 & 0 & 291.7 & 2.16E-03 & 0.9421 & 436 & Beta \\
\hline FTS & TDP & 2003 & 2 & 365.0 & 5.77E-03 & 2.9421 & 507 & Beta \\
\hline FTS & TDP & 2004 & 5 & 282.1 & 1.39E-02 & 5.9421 & 421 & Beta \\
\hline FTS & TDP & 2005 & 2 & 289.5 & 6.77E-03 & 2.9421 & 432 & Beta \\
\hline FTS & TDP & 2006 & 2 & 267.9 & 7.12E-03 & 2.9421 & 410 & Beta \\
\hline FTS & TDP & 2007 & 2 & 284.5 & 6.85E-03 & 2.9421 & 427 & Beta \\
\hline FTS & TDP & 2008 & 4 & 261.6 & 1.22E-02 & 4.9421 & 402 & Beta \\
\hline FTS & TDP & 2009 & 2 & 260.5 & 7.26E-03 & 2.9421 & 403 & Beta \\
\hline FTS & TDP & 2010 & 3 & 266.2 & 9.59E-03 & 3.9421 & 407 & Beta \\
\hline FTS & TDP & 2011 & 1 & 269.0 & 4.69E-03 & 1.9421 & 412 & Beta \\
\hline FTS & TDP & 2012 & 3 & 269.1 & 9.52E-03 & 3.9421 & 410 & Beta \\
\hline FTS & TDP & 2013 & 1 & 300.8 & 4.36E-03 & 1.9421 & 444 & Beta \\
\hline FTS & TDP & 2014 & 1 & 273.7 & 4.64E-03 & 1.9421 & 417 & Beta \\
\hline so & AOV & 1998 & 0 & 61320.0 & 1.29E-07 & 0.6801 & $5,272,320$ & Gamma \\
\hline SO & AOV & 1999 & 0 & 61320.0 & 1.29E-07 & 0.6801 & $5,272,320$ & Gamma \\
\hline SO & AOV & 2000 & 0 & 61320.0 & 1.29E-07 & 0.6801 & $5,272,320$ & Gamma \\
\hline So & AOV & 2001 & 0 & 61320.0 & 1.29E-07 & 0.6801 & $5,272,320$ & Gamma \\
\hline
\end{tabular}


Table 6. (continued).

\begin{tabular}{|c|c|c|c|c|c|c|c|c|}
\hline \multirow{2}{*}{$\begin{array}{l}\text { Failure } \\
\text { Mode }\end{array}$} & \multirow[b]{2}{*}{ Component } & \multirow[b]{2}{*}{ Year } & \multirow{2}{*}{$\begin{array}{l}\text { Number of } \\
\text { Failures }\end{array}$} & \multirow{2}{*}{$\begin{array}{l}\text { Demands/ } \\
\text { Run Hours }\end{array}$} & \multicolumn{4}{|c|}{ Bayesian Update } \\
\hline & & & & & Mean & Post A & Post B & Distribution \\
\hline so & AOV & 2002 & 0 & 61320.0 & 1.29E-07 & 0.6801 & $5,272,320$ & Gamma \\
\hline SO & AOV & 2003 & 0 & 61320.0 & $1.29 \mathrm{E}-07$ & 0.6801 & $5,272,320$ & Gamma \\
\hline SO & AOV & 2004 & 0 & 61320.0 & 1.29E-07 & 0.6801 & $5,272,320$ & Gamma \\
\hline SO & AOV & 2005 & 0 & 61320.0 & $1.29 \mathrm{E}-07$ & 0.6801 & $5,272,320$ & Gamma \\
\hline SO & AOV & 2006 & 0 & 61320.0 & $1.29 \mathrm{E}-07$ & 0.6801 & $5,272,320$ & Gamma \\
\hline SO & AOV & 2007 & 0 & 52560.0 & $1.29 \mathrm{E}-07$ & 0.6801 & $5,263,560$ & Gamma \\
\hline SO & AOV & 2008 & 0 & 52560.0 & $1.29 \mathrm{E}-07$ & 0.6801 & $5,263,560$ & Gamma \\
\hline so & AOV & 2009 & 0 & 52560.0 & $1.29 \mathrm{E}-07$ & 0.6801 & $5,263,560$ & Gamma \\
\hline SO & AOV & 2010 & 0 & 52560.0 & $1.29 \mathrm{E}-07$ & 0.6801 & $5,263,560$ & Gamma \\
\hline so & AOV & 2011 & 0 & 52560.0 & $1.29 \mathrm{E}-07$ & 0.6801 & $5,263,560$ & Gamma \\
\hline SO & AOV & 2012 & 0 & 52560.0 & $1.29 \mathrm{E}-07$ & 0.6801 & $5,263,560$ & Gamma \\
\hline SO & AOV & 2013 & 0 & 52560.0 & $1.29 \mathrm{E}-07$ & 0.6801 & $5,263,560$ & Gamma \\
\hline SO & AOV & 2014 & 0 & 52560.0 & $1.29 \mathrm{E}-07$ & 0.6801 & $5,263,560$ & Gamma \\
\hline SO & MOV & 1998 & 0 & 2522880.0 & 2.95E-08 & 0.5703 & $19,362,880$ & Gamma \\
\hline SO & MOV & 1999 & 0 & 2549160.0 & 2.94E-08 & 0.5703 & $19,389,160$ & Gamma \\
\hline SO & MOV & 2000 & 3 & 2575440.0 & $1.84 \mathrm{E}-07$ & 3.5703 & $19,415,440$ & Gamma \\
\hline so & MOV & 2001 & 0 & 2549160.0 & $2.94 \mathrm{E}-08$ & 0.5703 & $19,389,160$ & Gamma \\
\hline SO & MOV & 2002 & 1 & 2549160.0 & 8.10E-08 & 1.5703 & $19,389,160$ & Gamma \\
\hline so & MOV & 2003 & 0 & 2549160.0 & 2.94E-08 & 0.5703 & $19,389,160$ & Gamma \\
\hline SO & MOV & 2004 & 0 & 2549160.0 & $2.94 \mathrm{E}-08$ & 0.5703 & $19,389,160$ & Gamma \\
\hline SO & MOV & 2005 & 0 & 2549160.0 & 2.94E-08 & 0.5703 & $19,389,160$ & Gamma \\
\hline SO & MOV & 2006 & 0 & 2566680.0 & $2.94 \mathrm{E}-08$ & 0.5703 & $19,406,680$ & Gamma \\
\hline SO & MOV & 2007 & 0 & 2566680.0 & 2.94E-08 & 0.5703 & $19,406,680$ & Gamma \\
\hline SO & MOV & 2008 & 1 & 2566680.0 & 8.09E-08 & 1.5703 & $19,406,680$ & Gamma \\
\hline SO & MOV & 2009 & 1 & 2566680.0 & 8.09E-08 & 1.5703 & $19,406,680$ & Gamma \\
\hline SO & MOV & 2010 & 0 & 2654280.0 & 2.93E-08 & 0.5703 & $19,494,280$ & Gamma \\
\hline SO & MOV & 2011 & 0 & 2645520.0 & 2.93E-08 & 0.5703 & $19,485,520$ & Gamma \\
\hline SO & MOV & 2012 & 1 & 2592960.0 & 8.08E-08 & 1.5703 & $19,432,960$ & Gamma \\
\hline SO & MOV & 2013 & 3 & 2592960.0 & 1.84E-07 & 3.5703 & $19,432,960$ & Gamma \\
\hline so & MOV & 2014 & 1 & 2584200.0 & 8.08E-08 & 1.5703 & $19,424,200$ & Gamma \\
\hline
\end{tabular}


Table 7. Basic event UA trending data.

\begin{tabular}{ccccccccc}
\hline \multirow{2}{*}{$\begin{array}{c}\text { Failure } \\
\text { Mode }\end{array}$} & Component & Year & $\begin{array}{c}\text { UA } \\
\text { Hours }\end{array}$ & $\begin{array}{c}\text { Critical } \\
\text { Hours }\end{array}$ & Mean & Post A & Post B & Distribution \\
\hline UA & TDP & 1998 & 3132 & 154327 & $2.57 \mathrm{E}-02$ & 0.262 & 9.942 & Beta \\
UA & TDP & 1999 & 3320 & 227440 & $1.51 \mathrm{E}-02$ & 1.445 & 94.162 & Beta \\
\hline UA & TDP & 2000 & 2707 & 243437 & $1.12 \mathrm{E}-02$ & 3.050 & 270.128 & Beta \\
UA & TDP & 2001 & 2754 & 245992 & $1.14 \mathrm{E}-02$ & 1.046 & 90.747 & Beta \\
UA & TDP & 2002 & 2995 & 246143 & $1.23 \mathrm{E}-02$ & 2.009 & 161.619 & Beta \\
UA & TDP & 2003 & 2899 & 242006 & $1.21 \mathrm{E}-02$ & 2.183 & 178.378 & Beta \\
UA & TDP & 2004 & 2374 & 249750 & $9.51 \mathrm{E}-03$ & 1.837 & 191.385 & Beta \\
UA & TDP & 2005 & 1756 & 241269 & $7.45 \mathrm{E}-03$ & 2.428 & 323.270 & Beta \\
UA & TDP & 2006 & 2319 & 248995 & $9.26 \mathrm{E}-03$ & 2.391 & 255.670 & Beta \\
UA & TDP & 2007 & 2132 & 244139 & $8.71 \mathrm{E}-03$ & 0.725 & 82.469 & Beta \\
\hline UA & TDP & 2008 & 2499 & 246901 & $1.02 \mathrm{E}-02$ & 0.436 & 42.416 & Beta \\
UA & TDP & 2009 & 2848 & 241883 & $1.22 \mathrm{E}-02$ & 0.363 & 29.377 & Beta \\
UA & TDP & 2010 & 2401 & 247135 & $9.81 \mathrm{E}-03$ & 1.940 & 195.914 & Beta \\
UA & TDP & 2011 & 2461 & 238520 & $1.06 \mathrm{E}-02$ & 1.779 & 165.710 & Beta \\
UA & TDP & 2012 & 2848 & 242703 & $1.16 \mathrm{E}-02$ & 2.527 & 215.152 & Beta \\
UA & TDP & 2013 & 3915 & 236943 & $1.69 \mathrm{E}-02$ & 0.170 & 9.882 & Beta \\
UA & TDP & 2014 & 2066 & 248117 & $8.31 \mathrm{E}-03$ & 2.014 & 240.412 & Beta \\
\hline
\end{tabular}

Table 8. Failure mode acronyms.

\begin{tabular}{ll}
\multicolumn{1}{c}{ Failure Mode } & \multicolumn{1}{c}{ Failure Mode Description } \\
\hline FTLR & Fail to load/run \\
FTOC & Fail to open/close \\
FTOP & Fail to operate \\
FTR & Fail to run \\
FTR $<1$ H & Fail to run less than one hour \\
FTS & Fail to start \\
SO & Spurious operation \\
UA & Unavailability (maintenance or state of another component) \\
\hline
\end{tabular}


System Study

Reactor Core Isolation Cooling
2014 Update

December 2015 


\section{SYSTEM DESCRIPTION}

This is a generic description of the RCIC system. The models in SPAR incorporate plant-specific features that may not be described here.

The RCIC system is a single train standby system required by plant technical specifications for safe shut down of the plant. The system is not considered part of the emergency core cooling system, and does not have a loss-of-coolant accident function. The RCIC system is designed to ensure that sufficient reactor water inventory is maintained in the vessel to permit adequate core cooling. This prevents the reactor fuel from overheating in the event that one of the following occurs:

- A complete plant shut down occurs under conditions of a loss of the feedwater system before the reactor is depressurized to a point where the shutdown cooling system can be placed into operation

- The reactor pressure vessel is isolated in conjunction with a loss of coolant flow from the feedwater system

- A station blackout event occurs.

Following a normal reactor shut down, core fission product decay heat causes steam generation to continue, albeit at a reduced rate. During this time, the turbine bypass system diverts the steam to the main condenser, and the RCIC system supplies the makeup water required to maintain reactor vessel inventory. (Note that the RCIC system is just one of a number of systems capable of performing this function.) The turbine-driven pump supplies makeup water from the condensate storage tank (CST) to the reactor vessel. An alternate source of water is available from the suppression pool. The turbine is driven by a portion of the steam generated by the decay heat and exhausts to the suppression pool. This operation continues until the vessel pressure and temperature is reduced to the point that the residual heat removal system can be placed into operation.

The RCIC system is a single train standby system that contains a single $100 \%$ capacity steam turbinedriven pump. The RCIC system is capable of delivering reactor grade water from the CST to the reactor pressure vessel using reactor-decay-heat-generated steam as a source of energy to drive the turbine-driven pump. In the event that CST water is not available, an alternate source of water is available from the suppression pool. 19H19HFigure 4 provides a simplified diagram of a typical RCIC system.

The RCIC system steam turbines (at all plants) are Dresser-Rand Terry-Turbodyne (Terry) turbines designed for constant capacity over varying ranges of inlet steam pressure, typically $1040 \mathrm{psig}$ to 50 psig. These turbines have horsepower ratings that vary from 460 to 875 with associated pump flow rates from 400 to 800 gpm, depending on plant design. All Terry turbines that drive RCIC pumps use Woodward governors (type EG-M with EGR actuators) for speed control, including prevention of overspeed during "cold quick-starts." The ratings for the RCIC system varies by plant design class, with the older Design Class II plants having the smaller capacity systems and the newer Design Class VI plants having the higher capacity systems. However, because of the overall similarities of the system in the various design classes (same equipment manufacturer), no distinction was made in this report between the different design classes.

Turbine "cold quick-starts" are required to meet pump starting time limits in Safety Analysis Reports, and other requirements specified to meet the reactor safety analyses of the nuclear steam supply system vendor. A cold start is considered a start that occurs when a turbine has not been operated for at least 72 hours. Turbine "quick-starts" occur when the turbine is required to reach rated speed and pump flow in 30 to 120 seconds. Since standby turbines are idle for extended periods, lubricating oil drains from the turbine bearings, leaving the bearings vulnerable to excessive wear. Standby turbines supplied by Terry typically also use turbine lubricating oil as the hydraulic operating fluid for the governors and actuators. To provide bearing lubrication and governor oil on quick starts, a pressurized lubrication oil system is 
provided, which uses a shaft-driven lubrication oil pump. The shaft-driven pump provides lubrication oil to the turbine bearings and governor assembly as soon as the turbine begins to roll, enhancing both turbine lubrication and governor response.

To control turbine speed, a governor valve is provided for the turbine; the valve is typically supplied by Terry. The governor valve is fully open at the beginning of a quick-start and is designed to assume speed control during the startup when the turbine speed reaches the governor's minimum speed setting (approximately $2000 \mathrm{rpm}$ ). During a quick start the turbine steam admission valve opens fully and the turbine accelerates rapidly to the governor's minimum speed setting. At the minimum speed setting the governor starts to control turbine speed by throttling closed the governor valve. This limits the acceleration of the turbine to prevent an overspeed trip of the turbine. The closing of the governor valve slows the turbine to a speed less than the minimum speed. At this point the governor in conjunction with the ramp-generator increases turbine speed in a controlled manner to rated speed by slowly opening the governor valve. The ramp-generator controls and limits the time for the turbine to reach rated speed to approximately 30 seconds after the governor gains control. If inlet steam flow is excessive during a quick start, the governor valve cannot close sufficiently to limit speed before the turbine overspeeds.

The RCIC system instrumentation and control consists of system initiation and containment isolation circuitry. These two circuits provide different functions, both of which can contribute to system unreliability. The purpose of the initiation circuitry is to initiate actions (that is, start up the RCIC system) to ensure adequate core cooling when the reactor vessel is isolated from its primary heat sink and normal coolant makeup flow from the feedwater system is insufficient or unavailable. The purpose of the containment isolation circuitry is to initiate closure of appropriate containment isolation valves to limit fission product release should a RCIC steam line rupture occur.

The RCIC system initiation circuit allows for manual and automatic initiation of the system. Automatic initiation occurs for conditions of low reactor water level. The low reactor water level parameter is monitored by four transmitters that are connected to relays whose contacts are arranged in a one-out-of-two taken twice logic arrangement. Once initiated, the RCIC logic seals in and can be reset by the operator only when the reactor vessel level signals have cleared. Upon system initiation, the turbine steam supply valve opens to supply steam to the turbine, and the injection valve and the suction valve from the CST open to supply coolant flow to the reactor pressure vessel. In addition, the test-return line isolation valve is closed to allow full system flow and maintain primary containment isolation. Failure of any one of these valves to function during an initiation results in a failure of the system.

The RCIC system containment isolation circuitry typically provides automatic closure of the RCIC turbine steam supply isolation valves and turbine exhaust valve in the event of a steam line failure (high energy line break). The parameters monitored typically include high steam flow, low steam line pressure, high room delta-temperature, and high area temperature. Isolation of these valves disables the RCIC injection function: however, failure of this circuit to close these valves would not preclude operation of the system. During system standby a spurious isolation of the steam supply line caused by this circuit contributes to system unavailability, and during system operation the spurious isolation can contribute to system unreliability. 


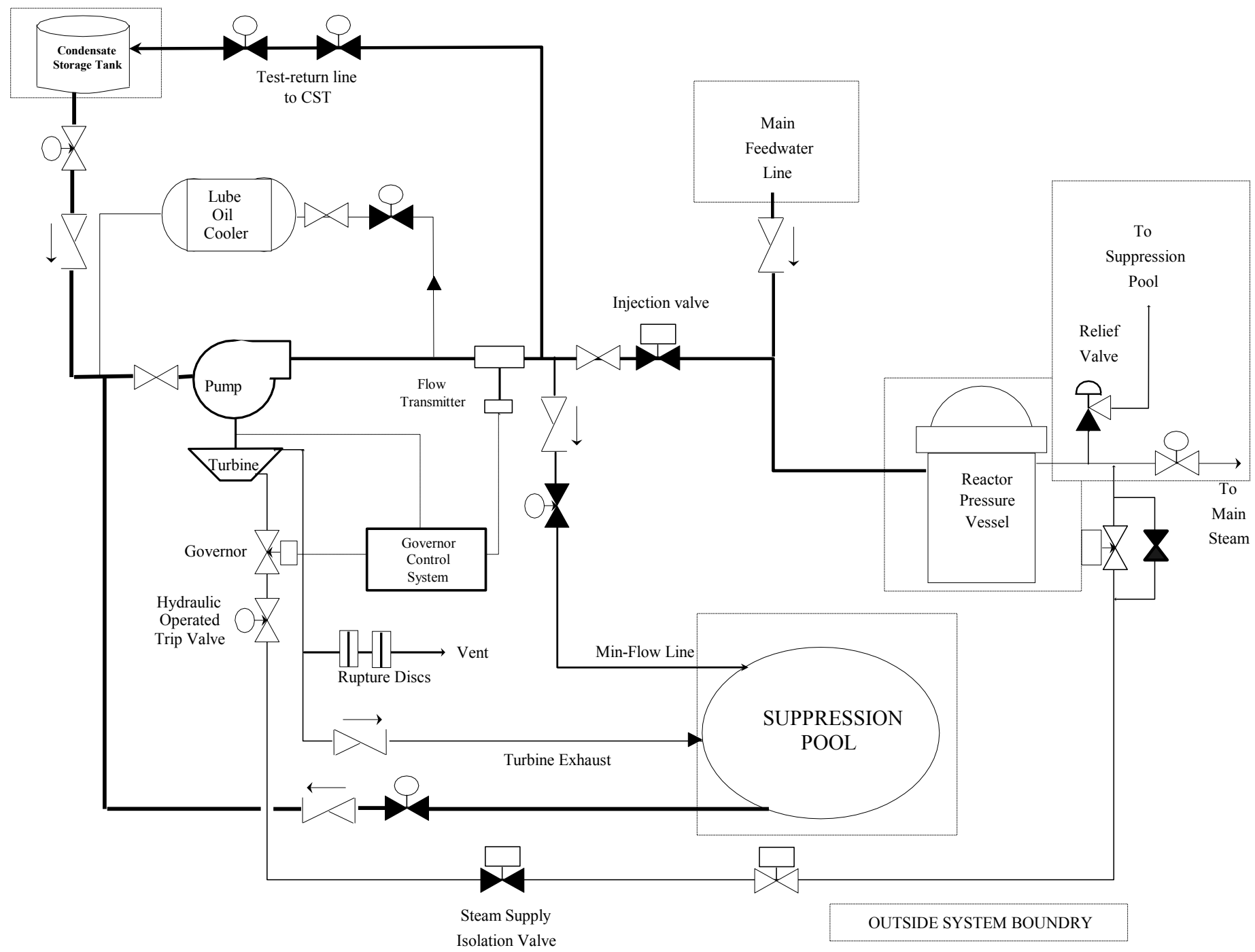

BWR Reactor Core Isolation Cooling

Figure 4. Simplified RCIC system schematic. 
System Study

Reactor Core Isolation Cooling
2014 Update December 2015 


\section{REFERENCES}

i. Nuclear Regulatory Commission, Component Reliability Data Sheets Update 2010, January 2012, http://nrcoe.inl.gov/resultsdb/publicdocs/AvgPerf/ComponentReliabilityDataSheets2010.pdf

ii. S.A. Eide et al., Industry-Average Performance for Components and Initiating Events at U.S. Commercial Nuclear Power Plants, Nuclear Regulatory Commission, NUREG/CR-6928, February 2007. 